\title{
Efficacy of Endoscopic Submucosal Dissection of Esophageal Neoplasms under General Anesthesia
}

\author{
Koichi Hamada ${ }^{1,2}$, Koichiro Kawano ${ }^{3}$, Atsushi Yamauchi ${ }^{4}$, Ryota Koyanagi ${ }^{1}, 2$, Yoshinori Horikawa ${ }^{1}$, Shinya Nishida ${ }^{1}$, Yoshiki Shiwa ${ }^{1}$, \\ Noriyuki Nishino ${ }^{1}$ and Michitaka Honda ${ }^{2,5}$ \\ ${ }^{1}$ Department of Gastroenterology, Southern-Tohoku General Hospital, Koriyama, ${ }^{2}$ Department of Minimally Invasive Surgical and Medical \\ Oncology, Fukushima Medical University, Fukushima, ${ }^{3}$ Department of Gastroenterology, Hyogo Prefectural Awaji Medical Center, Sumoto, \\ ${ }^{4}$ Department of Gastroenterology, Kitano Hospital, Osaka, ${ }^{5}$ Department of Surgery, Southern-Tohoku General Hospital, Koriyama, Japan
}

Background/Aims: Evidence that general anesthesia (GA) reduces the operative time of esophageal endoscopic submucosal dissection (ESD) is currently insufficient. This study aims to evaluate the efficacy and safety of esophageal ESD under GA.

Methods: A total of 227 lesions from 198 consecutive patients with superficial esophageal neoplasms treated by ESD at 3 Japanese institutions between April 2011 and September 2017 were included in this retrospective study. For ESD, GA and deep sedation (DS) were used in 102 (51.5\%, GA group) and 96 patients (48.5\%, DS group), respectively.

Results: There were no statistically significant differences in age, sex, or comorbidities between the groups. In the GA group, the tumor size was larger (21 [3-77] mm vs. 14 [3-63] mm, $p<0.001)$, luminal circumference was larger $(\geq 2 / 3 ; 13.9 \%$ vs. $5.4 \%$, $p=0.042)$, procedure time was shorter (28 [5-202] min vs. 40 [8-249] $\mathrm{min}, p<0.001)$, and submucosal dissection speed was faster $\left(25.2[7.8-157.2] \mathrm{mm}^{2} / \mathrm{min}^{2}\right.$ vs. $\left.16.2[2.4-41.3] \mathrm{mm}^{2} / \mathrm{min}, p<0.001\right)$. The rates of intraoperative perforation and aspiration pneumonia were lower in the GA group, but the difference did not achieve statistical significance $(p=0.242$ and $p=0.242)$.

Conclusions: GA shortens the procedure time of esophageal ESD. Clin Endosc 2019;52:252-257

Key Words: Esophageal cancer; Endoscopic submucosal dissection; Anesthesia

\section{INTRODUCTION}

Endoscopic resection is being more frequently performed as an esophageal-sparing and minimally invasive treatment for superficial esophageal neoplasia. ${ }^{1,2}$ Endoscopic treatments have shown acceptable oncological outcomes, with a local recurrence rate reported to range from $0.3 \%$ to $11.5 \%{ }^{3}$ In addition, recent technical advances in endoscopic submucosal

Received: August 29, 2018 Revised: October 31, 2018

Accepted: November 23, 2018

Correspondence: Koichi Hamada

Department of Gastroenterology, Southern-Tohoku General Hospital, 7-115, Yatsuyamada, Koriyama-shi, Fukushima 963-8563, Japan

Tel: +81-024-934-5322, Fax: +81-024-934-5328,

E-mail: koichi.hamada@mt.strins.or.jp

ORCID: https://orcid.org/0000-0002-4669-5501

(cc) This is an Open Access article distributed under the terms of the Creative Commons Attribution Non-Commercial License (http://creativecommons.org/ licenses/by-nc/3.0) which permits unrestricted non-commercial use, distribution, and reproduction in any medium, provided the original work is properly cited. dissection (ESD) are expected to further improve the survival of patients receiving such treatment by allowing for en bloc resection of lesions. ${ }^{4,5}$

Reducing the procedure time is the current major challenge in esophageal ESD. Esophageal squamous cell carcinoma progresses by intraepithelial spread, which requires extensive resection. Moreover, the operability of the endoscope is more limited in the esophagus than in the stomach, and perforation is more likely to cause a severe condition in the esophagus. Therefore, very careful endoscopic manipulation is required, necessitating a longer operative time. Causes of perforation include excessive insufflation or direct trauma to the muscle layer induced by energy devices. Since the thickness of the dilated esophageal wall during insufflation is only 3 to $4 \mathrm{~mm}$, slight injury to the deeper layer can induce perforation; the patient's respiration or physical movements during the operation may also increase the risk of perforation.

Therefore, controlling body movements and respiratory 
fluctuations may help prevent this complication and shorten the procedure time. The effectiveness of anesthesia using propofol during esophageal ESD has been reported in previous studies, ${ }^{6-8}$ and the combination of propofol and dexmedetomidine hydrochloride represents a promising strategy for mitigating the risk of ESD-related complications and shortening the procedure time.' Some studies have shown the effectiveness of general anesthesia (GA) during gastric ESD; ${ }^{10-}$

${ }^{13}$ however, no studies have described whether or not GA reduces the operative time in esophageal ESD.

We conducted a multi-center retrospective cohort study to investigate the hypothesis that esophageal ESD under GA would shorten the procedure time compared to ESD under deep sedation (DS) using midazolam and pentazocine. We also evaluated the incidence of complications. This study aimed to provide beneficial information to enable clinical physicians to perform esophageal ESD more safely.

\section{MATERIALS AND METHODS}

\section{Patients}

This study had a three-center, retrospective cohort design. A total of 227 lesions in 198 consecutive patients who underwent ESD for superficial esophageal neoplasia at Southern-Tohoku General Hospital in Fukushima, Hyogo Prefectural Awaji Medical Center in Hyogo Prefecture, and Kitano Hospital in Osaka between April 2011 and November 2017 were included in the study.

\section{Outcomes and survey items}

The following outcomes were compared between patients who underwent the procedure under GA and those who received DS: the primary outcome was the ESD procedure time, and the secondary outcomes were the complications associated with ESD, i.e., perforation, postoperative hemorrhaging, and aspiration pneumonia, and complications associated with anesthesia. In addition, we isolated the following potential confounding factors from electrical medical records to secure the validity of the comparison: age, sex, body mass index (BMI), American Society of Anesthesiologists (ASA) physical status classification, ${ }^{14}$ site, diameter, and circumference of the lesion; resected diameter and circumference of the lumen; dissection speed; en bloc resection rate; number of devices used; and rate of use of the clip-with-line method. In the DS group, we evaluated the rate of unintended DS. Systolic blood pressure $<90 \mathrm{~mm} \mathrm{Hg}$ was defined as hypotension, heart rate $<50$ beats/min as bradycardia, respiratory rate $<8$ breaths/min as bradypnea, and $\mathrm{SpO}_{2}<90 \%$ as hypoxemia.

This study was approved by the ethics committees of all participating institutions and conducted in accordance with the 1975 Declaration of Helsinki.

\section{ESD procedure}

ESD was performed using a single-channel endoscope with a water jet system (GIF-Q260J; Olympus Medical Systems Co., Tokyo, Japan), with $\mathrm{CO}_{2}$ insufflation (UCR; Olympus Medical Systems). A Dual knife (KD-650Q; Olympus Medical Systems) was used for the procedure, and a Hook knife (KD260LR; Olympus Medical Systems) was added as needed.

Sodium hyaluronate (0.4\%, MucoUp; Seikagaku Co., Tokyo, Japan) was locally injected into the submucosa using a $25-\mathrm{G}$ injection needle (Needle Master; Olympus Medical Systems). Hemostatic graspers (Coagrasper; FD-411QR; Olympus Medical Systems) were used for preventive coagulation and intraoperative hemostasis of vessels. A VIO300D (ERBE Elektromedizin Gmbh, Tübingen, Germany) was used as the electrosurgery unit. In this study, all of the surgeons in each facility were Certified Surgeons of the Japanese Gastroenterological Endoscopy Society, each with experience performing more than 30 cases of esophageal ESD and more than 100 cases of gastric ESD.

The procedure time was defined as the time from the beginning of the submucosal injection to complete removal. The average dissection speed was calculated by dividing the time of the procedure by the area of the resected specimen, which was calculated as $3.14 \times 0.25 \times$ long axis $\times$ minor axis. The procedure time included delays due to movement of the patient during the procedure.

Esophageal perforation was defined as macroscopic perforation to the esophageal wall, and postoperative hemorrhaging was defined as post-ESD hematemesis requiring an endoscopic procedure, bleeding accompanied by melena, anemia (decrease of $\geq$ hemoglobin $2 \mathrm{~g} / \mathrm{dL}$ ), or a condition requiring transfusion.

\section{Deep sedation and general anesthesia}

In this study period, all patients were allocated to receive DS or GA during the operation, at the physician's discretion.

The principal of DS was established by the ASA. ${ }^{14}$ In brief, an initial dose of $7.5 \mathrm{mg}$ pentazocine and $2 \mathrm{mg}$ midazolam for patients weighing $<50 \mathrm{~kg}$ or $3 \mathrm{mg}$ midazolam for patients weighing $\geq 50 \mathrm{~kg}$ was administered. In response to patients' body movements, $1 \mathrm{mg}$ midazolam or $7.5 \mathrm{mg}$ pentazocine was additionally administered. After completing ESD, $0.5 \mathrm{mg}$ flumazenil was administered to awaken the patients.

In the GA group, a propofol intravenous drip of 1-2 mg/ $\mathrm{kg} / \mathrm{min}$ was administered, $3 \%$ sevoflurane was administered for tracheal intubation, and $0.6-0.9 \mathrm{mg} / \mathrm{kg}$ rocuronium was administered as a muscle relaxant. Intraoperative sevoflurane 
was maintained at $1 \%-1.5 \%$, and $0.3-0.5 \mu \mathrm{g} / \mathrm{kg} / \mathrm{min}$ remifentanil was administered as an adjuvant.

All agents were administered by a gastroenterologist who was not involved in the ESD procedure. Oxygen at $2 \mathrm{~L} / \mathrm{min}$ was administered via a nasal cannula in the endoscopy room, and 3-lead electrocardiography, pulse oximetry, and automatic blood pressure monitoring were performed to monitor vital signs and oxygen saturation, with recordings made every 5 $\min$.

\section{Statistical analyses}

The chi-squared test, Kruskal-Wallis exact test, or Fisher's exact test were used to compare categorical variables. Continuous variables were expressed as median and range. Median values of continuous data were compared using the Mann-Whitney $U$-test. All statistical analyses were performed using the IBM SPSS software program (IBM Co., Armonk, NY, USA). All tests were two-tailed, and a $p$-value of $<0.05$ was considered statistically significant.

\section{RESULTS}

\section{Patient characteristics}

Patient characteristics are summarized in Table 1. Among 198 patients with esophageal neoplasia, ESD under GA was performed in 102 patients (51.5\%), and ESD under DS was performed in 96 patients (48.5\%). There were no significant differences in age, sex, BMI, comorbidities, or use of antithrombotic drugs between the GA and DS groups. Patients with a poorer ASA score were more common in the GA group than in the DS group.

\section{Clinicopathological factors between the GA and DS group}

There were significant differences in the size of tumors $(p<0.001)$, tumor circumference of the lumen $(p=0.042)$, and histologic type $(p=0.030)$ between the groups. There were no significant differences in the location of tumors, depth of invasion, or lymphatic and vascular invasion (Table 2). Although there was no significant difference between the 2 groups in terms of the resected diameter, the GA group had a significantly higher proportion of patients with a lumen circumference $\geq 2 / 3$ ( $p=0.005$ ).

\section{Procedure outcomes}

Table 3 shows the outcomes. The ESD procedure times for the GA and DS groups were 28 (5-202) and 40 (8-249) min, respectively $(p<0.001)$, indicating a significantly shorter procedure time in the GA group. There were no significant differences in the incidence of complications between the 2 groups. In the DS group, intraoperative perforation occurred in 2 pa-

Table 1. Baseline Characteristics of the Patients in the Two Groups

\begin{tabular}{|c|c|c|c|}
\hline & General anesthesia $(n=102)$ & Deep sedation $(n=96)$ & $p$-value \\
\hline Age (yr) & $70(50-87)$ & $69(43-82)$ & 0.183 \\
\hline Sex & & & $>0.999$ \\
\hline Male & $89(87.3)$ & $83(86.5)$ & \\
\hline Female & $13(12.7)$ & $13(13.5)$ & \\
\hline BMI $\left(\mathrm{kg} / \mathrm{m}^{2}\right)$ & $22.5(11.3-28.1)$ & $21.3(14.6-32.3)$ & 0.397 \\
\hline ASA score & & & 0.018 \\
\hline $\mathrm{I}$ & $30(29.4)$ & $19(19.8)$ & \\
\hline II & $67(65.7)$ & $77(80.2)$ & \\
\hline III & $5(4.9)$ & 0 & \\
\hline \multicolumn{4}{|l|}{ Comorbidity } \\
\hline Hypertension & $52(51.0)$ & $45(46.9)$ & 0.573 \\
\hline Hyperlipidemia & $13(12.7)$ & $15(15.6)$ & 0.684 \\
\hline Diabetes mellitus & $15(14.7)$ & $13(13.5)$ & 0.841 \\
\hline Chronic kidney diseases & $3(2.9)$ & 0 & 0.247 \\
\hline Chronic liver diseases & $4(3.9)$ & $9(9.4)$ & 0.155 \\
\hline Antithrombotic drugs & $26(25.5)$ & $19(19.8)$ & 0.397 \\
\hline
\end{tabular}

Values are expressed as median (range) or $n(\%)$.

ASA, American Society of Anesthesiologists; BMI, body mass index. 
tients (1.8\%) and aspiration pneumonia occurred in 2 patients $(1.8 \%)$. The perforation in 1 patient was not from trauma to the muscle layer caused by the knife but rather from a large rupture caused by intraoperative eructation. As the perforation was large and could not be sutured with clips, surgical drainage was performed. The other patient's perforation was induced by trauma to the muscle layer caused by a knife and was successfully sutured with clips. There were no marked differences in the en bloc resection rates between the 2 groups. The dissection speed was significantly higher in the GA group than in the DS group $(p<0.001)$. The number of patients who required the use of multiple devices was significantly higher in the DS group than in the GA group ( $p=0.001)$, but the rate of use of the clip-with-line method ${ }^{15,16}$ was comparable between the 2 groups.

In the DS group, the median total amount of midazolam was 10.8 (4.0-27.0) $\mathrm{mg}$ and the median total amount of pentazocine was $7.5(0-30) \mathrm{mg}$. The median number and amount of bolus injections of midazolam were $2(0-12)$ times and 2.0 $(0-12) \mathrm{mg}$, respectively. The median number and amount of bolus injections of pentazocine were $0(0-3)$ times and $0(0-22.5) \mathrm{mg}$, respectively. The median frequency of body movement during ESD was 2 (0-14) times.

\section{Safety evaluation}

There were no complications associated with GA and no mortalities among the enrolled patients. In the DS group, hypotension occurred in 7 patients (6.3\%), bradycardia occurred in 5 patients (4.5\%), hypoxemia occurred in 2 patients (1.8\%), and bradypnea occurred in 3 patients (2.7\%). No patients required assisted mask ventilation or intubation. A paradoxical response during sedation occurred in 3 patients (2.7\%), but ESD was successfully completed under DS.

\section{DISCUSSION}

In the present study, we compared the operative time and incidence of complications between the patients who underwent esophageal ESD under GA and those who received DS. Our results yielded 2 important findings. One is that the operative time of esophageal ESD with GA was shorter than that of ESD with DS. The other is that there were no significant differences in the incidence of all types of complications

Table 2. Clinicopathological Factors between General Anesthesia and Deep Sedation

\begin{tabular}{|c|c|c|c|}
\hline & General anesthesia $(n=115)$ & Deep sedation $(n=112)$ & $p$-value \\
\hline Size of tumors $(\mathrm{mm})$ & $21(3-77)$ & $14(3-63)$ & $<0.001$ \\
\hline Location of tumors & & & 0.05 \\
\hline Upper third & $22(19.1)$ & $11(9.8)$ & \\
\hline Middle third & $67(58.3)$ & $63(56.3)$ & \\
\hline Lower third & $26(22.6)$ & $38(33.9)$ & \\
\hline Circumference of the lumen (tumor) & & & 0.042 \\
\hline$<2 / 3$ & $99(86.1)$ & $106(94.6)$ & \\
\hline$\geq 2 / 3$ & $16(13.9)$ & $6(5.4)$ & \\
\hline Histologic type & & & 0.03 \\
\hline Squamous cell carcinoma & $98(85.2)$ & $108(96.4)$ & \\
\hline Intraepithelial neoplasia & $9(7.8)$ & $3(2.7)$ & \\
\hline Adenocarcinoma & $7(6.1)$ & $1(0.9)$ & \\
\hline Others & $1(0.9)$ & 0 & \\
\hline Depth of invasion & & & 0.105 \\
\hline $\mathrm{EP}$ & $57(49.6)$ & $47(42.0)$ & \\
\hline LPM & $25(21.7)$ & $40(35.7)$ & \\
\hline MM & $19(16.5)$ & $17(15.2)$ & \\
\hline SM & $14(12.2)$ & $8(7.1)$ & \\
\hline Lymphatic invasion & $5(4.3)$ & $2(1.8)$ & 0.446 \\
\hline Vascular invasion & $3(2.6)$ & 0 & 0.247 \\
\hline
\end{tabular}

Values are expressed as median (range) or $n(\%)$.

EP, epithelium; LPM, lamina propria mucosae; MM, muscularis mucosae; SM, submucosa. 
Table 3. Procedure Outcomes and Adverse Events

\begin{tabular}{|c|c|c|c|}
\hline & General anesthesia $(n=115)$ & Deep sedation $(n=112)$ & $p$-value \\
\hline Size of resected specimen, (mm) & $35(12-90)$ & $32(10-77)$ & 0.129 \\
\hline Circumference of resected ulcer of the lumen & & & 0.005 \\
\hline$<2 / 3$ & $86(74.8)$ & $96(85.7)$ & \\
\hline$\geq 2 / 3$ & $29(25.2)$ & $16(14.3)$ & \\
\hline En bloc resection rate & $115(100)$ & $110(98.2)$ & 0.242 \\
\hline Procedure time (min) & $28(5-202)$ & $40(8-249)$ & $<0.001$ \\
\hline Submucosal dissection speed $\left(\mathrm{mm}^{2} / \mathrm{min}\right)$ & $25.2(7.8-157.2)$ & $16.2(2.4-41.3)$ & $<0.001$ \\
\hline Single device & $115(100)$ & $102(91.1)$ & 0.001 \\
\hline Clip-with-line method & $19(16.5)$ & $28(25.0)$ & 0.141 \\
\hline Delayed bleeding & $2(1.7)$ & 0 & 0.498 \\
\hline Intraoperative perforation & 0 & $2(1.8)$ & 0.242 \\
\hline Aspiration pneumonia & 0 & $2(1.8)$ & 0.242 \\
\hline Stenosis & $9(7.8)$ & $8(7.1)$ & 1 \\
\hline Odontoclasis & 0 & 0 & - \\
\hline Anaphylaxis & 0 & 0 & - \\
\hline Thromboembolism & 0 & 0 & - \\
\hline Tracheobronchial injury & 0 & 0 & - \\
\hline Mortality & 0 & 0 & - \\
\hline
\end{tabular}

Values are expressed as median (range) or $n(\%)$.

related to ESD or sedation between the 2 groups. The GA group had a higher proportion of patients with a larger lumen circumference of the lesion. Therefore, these results suggest that operators tend to choose GA when treating difficult cases. Even though difficult cases were more common in the GA group, the procedure time in the GA group was ultimately shorter than that in the DS group, and the dissection speed was faster as well. This reflects the effect of a reduced procedure time by GA. In addition, there were no complications associated with GA among the enrolled patients.

Among several reports that have described the methods of anesthesia for esophageal ESD, one study reported that the combination of propofol and dexmedetomidine hydrochloride shortened the esophageal ESD procedure time significantly compared to midazolam or flunitrazepam, ${ }^{9}$ as this combination was able to suppress the patients' body movements during the procedure. The major point of emphasis in the present study is that GA can completely suppress patients' body movements and stabilize their respiratory fluctuations and heartbeat. We believe that these effects of GA represent a significant advantage with regard to ensuring the safety of esophageal ESD.

However, another previous study reported results contrary to those of our study, with the procedure time on using nonGA being shorter than that of procedures using GA. ${ }^{13}$ That study also reported that the proportion of non-expert operators performing ESD under GA was significantly higher than that of those performing ESD under DS, which may represent a major confounding factor in their results. In the present study, interoperator differences were controlled, as all of the surgeons in this study were experts with experience in managing over 100 cases of gastric ESD and 30 cases of esophageal ESD, thereby precluding such confounding.

There were no significant differences between the 2 groups in terms of the 3 complications of perforations, hemorrhaging, and aspiration pneumonia, which were the secondary outcomes of this study. This result may be due to the low incidence of complications overall (GA group: 2 patients; DS group: 4 patients) and the small sample size. Indeed, the higher number of perforations in the DS group could have been prevented by controlling body movements, respiratory changes, and eructation. Aspiration pneumonia occurred in the DS group. Previous reports have also indicated that gastric ESD under conscious sedation is a risk factor for pulmonary aspiration; ${ }^{17,18}$ therefore, airway management and positive-pressure ventilation may be effective measures for preventing these complications. Conversely, there were more patients with postoperative hemorrhaging in the GA group than in the DS group. However, analysis according to the patients' background characteristics suggested that the GA group was at 
higher risk of hemorrhaging simply because they had higher numbers of large lesions and required a larger extent of resection.

One of the limitations of this study was the determination of the outcomes. Complications in esophageal ESD are quite rare, with rates of $0 \%-6.9 \%$ for intraoperative perforations and $1.6 \%-6.6 \%$ for aspiration pneumonia. ${ }^{1,2,4,5,8,19}$ If we had selected the complications as the primary outcome, the analyses would have had insufficient statistical power. Therefore, we set the operative time as a surrogate endpoint in this study. Another associated limitation is that the allocation of patients to the GA or DS groups was decided at the physicians' discretion, so there may be some residual confounding. However, as mentioned above, given that the GA group included more patients with more difficult-to-treat lesions, the results demonstrating a shortened procedure time in the GA group with no marked differences in complications are decidedly noteworthy. Further studies are needed to compare dexmedetomidine or propofol with GA. In addition, future studies should evaluate the satisfaction among endoscopists and patients according to the sedation method and the speed of recovery.

In conclusion, esophageal ESD under GA is associated with a shortened ESD procedure time compared to ESD under DS.

\section{Conflicts of Interest}

The authors have no financial conflicts of interest.

\section{Acknowledgements}

We thank Japan Medical Communication (http://www.japan-mc.co.jp/) for the English language editing.

\section{Author Contributions}

Conceptualization: Koichi Hamada, Koichiro Kawano

Data curation: KH, KK, Atsushi Yamauchi, Ryota Koyanagi, Yoshinori Horikawa, Yoshiki Shiwa

Formal analysis: KH, Shinya Nishida

Investigation: $\mathrm{KH}, \mathrm{KK}$

Methodology: KH, KK, Michitaka Honda

Project administration: KH, KK

Supervision: Noriyuki Nishino

Writing-original draft: $\mathrm{KH}$

Writing-review\&editing: $\mathrm{KK}, \mathrm{MH}$

\section{REFERENCES}

1. Oyama T, Tomori A, Hotta K, et al. Endoscopic submucosal dissection of early esophageal cancer. Clin Gastroenterol Hepatol 2005;3(7 Suppl 1):S67-S70.
2. Fujishiro M, Yahagi N, Kakushima N, et al. Endoscopic submucosal dissection of esophageal squamous cell neoplasms. Clin Gastroenterol Hepatol 2006;4:688-694.

3. Guo HM, Zhang XQ, Chen M, Huang SL, Zou XP. Endoscopic submucosal dissection vs endoscopic mucosal resection for superficial esophageal cancer. World J Gastroenterol 2014;20:5540-5547.

4. Ishihara R, Iishi H, Uedo N, et al. Comparison of EMR and endoscopic submucosal dissection for en bloc resection of early esophageal cancers in Japan. Gastrointest Endosc 2008;68:1066-1072.

5. Takahashi H, Arimura Y, Masao H, et al. Endoscopic submucosal dissection is superior to conventional endoscopic resection as a curative treatment for early squamous cell carcinoma of the esophagus (with video). Gastrointest Endosc 2010;72:255-264, 264.e1-e2.

6. Nonaka S, Kawaguchi Y, Oda I, et al. Safety and effectiveness of propofol-based monitored anesthesia care without intubation during endoscopic submucosal dissection for early gastric and esophageal cancers. Dig Endosc 2015;27:665-673.

7. Gotoda T, Okada H, Hori K, et al. Propofol sedation with a target-controlled infusion pump and bispectral index monitoring system in elderly patients during a complex upper endoscopy procedure. Gastrointest Endosc 2016;83:756-764.

8. Ominami M, Nagami Y, Shiba M, et al. Comparison of propofol with midazolam in endoscopic submucosal dissection for esophageal squamous cell carcinoma: a randomized controlled trial. J Gastroenterol 2018;53:397-406.

9. Nonaka T, Inamori M, Miyashita T, et al. Feasibility of deep sedation with a combination of propofol and dexmedetomidine hydrochloride for esophageal endoscopic submucosal dissection. Dig Endosc 2016;28:145-151.

10. Yurtlu DA, Aslan F, Ayvat P, et al. Propofol-based sedation versus general anesthesia for endoscopic submucosal dissection. Medicine (Baltimore) 2016;95:e3680.

11. Rong QH, Zhao GL, Xie JP, Wang LX. Feasibility and safety of endoscopic submucosal dissection of esophageal or gastric carcinomas under general anesthesia. Med Princ Pract 2013;22:280-284.

12. Yamashita K, Shiwaku H, Ohmiya T, et al. Efficacy and safety of endoscopic submucosal dissection under general anesthesia. World J Gastrointest Endosc 2016;8:466-471.

13. Yagi Kuwata N, Gotoda T, Suzuki S, Mukai S, Itoi T, Moriyasu F. Reasonable decision of anesthesia methods in patients who underwent endoscopic submucosal dissection for superficial esophageal carcinoma: a retrospective analysis in a single Japanese institution. Turk J Gastroenterol 2016;27:91-96.

14. American Society of Anesthesiologists Task Force on Sedation and Analgesia by Non-Anesthesiologists. Practice guidelines for sedation and analgesia by non-anesthesiologists. Anesthesiology 2002;96:1004-1017.

15. Ota M, Nakamura T, Hayashi K, et al. Usefulness of clip traction in the early phase of esophageal endoscopic submucosal dissection. Dig Endosc 2012;24:315-318.

16. Oyama T. Counter traction makes endoscopic submucosal dissection easier. Clin Endosc 2012;45:375-378.

17. Watari J, Tomita T, Toyoshima F, et al. The incidence of "silent" free air and aspiration pneumonia detected by CT after gastric endoscopic submucosal dissection. Gastrointest Endosc 2012;76:1116-1123.

18. Park CH, Kim H, Kang YA, et al. Risk factors and prognosis of pulmonary complications after endoscopic submucosal dissection for gastric neoplasia. Dig Dis Sci 2013;58:540-546.

19. Tsujii Y, Nishida T, Nishiyama O, et al. Clinical outcomes of endoscopic submucosal dissection for superficial esophageal neoplasms: a multicenter retrospective cohort study. Endoscopy 2015;47:775-783. 